\title{
Alcohol septal ablation in hypertrophic obstructive cardiomyopathy: a case with a very high left ventricular outflow tract gradient
}

\begin{abstract}
For more than 20 years, alcohol septal ablation (ASA) has shown to be a safe and effective procedure in the treatment of hypertrophic obstructive cardiomyopathy (HOCM), with results similar to those of surgical myectomy. To our knowledge, we present the first documented case of a HOCM with a very high left ventricular outflow tract (LVOT) gradient $(>250 \mathrm{~mm} \mathrm{Hg}$ ) managed with ASA, with a decrease of $>50 \%$ of gradient after the procedure.
\end{abstract}

Keywords: ablation, cardiomyopathy, hypertrophic, left ventricular outflow gradient, catheter, genetic disorder, hypertrophic obstructive cardiomyopathy, betamyosin heavy chain, syncope
Volume 3 Issue I - 2019

\author{
Jose Pascual Salas Llamas,' Efrain Gaxiola \\ López, ${ }^{2}$ Yara Itzel Tejeda Vargas, ${ }^{3}$ Alan Mendez \\ Ruiz ${ }^{4}$ \\ 'MD, Hospital Ángeles del Carmen, Unidad de Cardiología, \\ Tarascos 3473, Piso 6, 446670, Guadalajara, Jalisco, México \\ 2MD, Hospital Ángeles del Carmen, Unidad de Cardiología, \\ Centro Especializado en Terapia Endovascular (CETEN), Colonia \\ Monraz 44670, Guadalajara Jalisco, Mexico \\ ${ }^{3}$ Hospital Ángeles del Carmen, Unidad de Cardiología, Tarascos \\ 3473, Piso 6, 446670, Guadalajara, Jalisco, México \\ ${ }^{4}$ Hospital Ángeles del Carmen, Unidad de Cardiología, Tarascos \\ 3473, Piso 6, 446670, Guadalajara, Jalisco, México
}

Correspondence: Jose Pascual Salas Llamas, Hospital Ángeles del Carmen, Unidad de Cardiología, Tarascos 3473, Piso 6, 446670, Guadalajara, Jalisco, Mexico, Tel 3317948836, Email doc.salas@hotmail.com, consultacardio610@gmail.com

Received: January 15, 2019 | Published: February 04, 2019
Abbreviations: HCM, hypertrophic cardiomyopathy; MYH7, beta-myosin heavy chain, MYBPC3, myosin-binding protein $\mathrm{C}$; HOCM, hypertrophic obstructive cardiomyopathy; ASA, alcohol septal ablation; LVOT, left ventricular outflow tract; SAM, systolic anterior motion; OTW, over-the-wire; ESC, European society of cardiology; NYHA I, NYHA functional class

\section{Introduction}

Hypertrophic cardiomyopathy (HCM) is a genetic disorder of the cardiac muscle characterized by left ventricular hypertrophy, myofibrillar disarray and myocardial stiffness. This entity affects approximately 1:500 individuals and is the most common cause of sudden death in young athletes. ${ }^{1}$ Approximately $40 \%$ of the cases of HCM are associated with mutations in genes that encode for beta-myosin heavy chain (MYH7) and myosin-binding protein $\mathrm{C}$ (MYBPC3). ${ }^{2}$ The majority of hypertrophic obstructive cardiomyopathy (HOCM) patients are detected incidentally; dyspnea is the frequent symptom in $90 \%$ of the cases followed by chest pain in a $70-80 \%$ and less frequently syncope in $20 \%$, or to debut with sudden death. ${ }^{2}$ Currently, septal reduction therapy for patients with HCM can be done either by surgical myectomy or by alcohol septal ablation (ASA). ${ }^{3}$ Surgical myectomy is the most commonly performed surgical procedure, in patients with a resting or maximum provoked left ventricular outflow tract (LVOT) gradient of $\geq 50 \mathrm{~mm} \mathrm{Hg}$, who are in NYHA functional Class III-IV, despite maximum tolerated medical therapy. ${ }^{2}$ ASA was introduced as a percutaneous alternative to surgical myectomy ${ }^{3}$ with the first case reported by Dr. Ulrich Sigwart published in 1995, and subsequently in North America in 1996. ${ }^{4}$ Since then, it has shown results similar to that of surgical myectomy. To our concern, we present the first clinical case of a very high LVOT gradient $(>250 \mathrm{~mm} \mathrm{Hg}) \mathrm{HCM}$ treated with ASA with significant reduction $>50 \%$ of gradient after procedure.

\section{Case Series}

65 -year-old male patient with a history of systemic arterial hypertension; diagnosis of asymmetric HOCM of 3 years of evolution described by echocardiography as LV hypertrophy with a basal distribution, septum width of $18 \mathrm{~mm}$, LVOT maximum gradient of $55 \mathrm{~mm} \mathrm{Hg}$ at rest, LVEF 75\%; managed with ACE inhibitor and betablockers. He was seen in consultation due to symptoms of dyspnea, palpitations and progressive angina, NYHA functional class II-III. A new transthoracic echocardiogram was performed with LVOT obstructive gradient of $65 \mathrm{mmHg}$ (Figure 1), $21 \mathrm{~mm}$ septum with, LVEF 76\%, moderate dilation of left atrium, PASP $32 \mathrm{~mm} \mathrm{Hg}$; EKG Sokolow-Lyon index $35 \mathrm{~mm}$. Dobutamine stress echocardiogram was performed with dynamic obstructive gradient of $257 \mathrm{~mm} \mathrm{Hg}$, moderate-severe systolic anterior motion (SAM) of mitral valve. Next, cardiac catheterization and ventriculography were performed observing coronary arteries without significant lesions and adequate diameter of septal branches, severely hypertrophied LV with LVEF $80 \%$ and end-diastolic pressure of $16 \mathrm{~mm} \mathrm{Hg}$. We decided to perform ASA.

Technique: Bilateral femoral arterial accesses were made and a pigtail catheter was placed in LV for pressure assessment during the procedure. Through right femoral artery, the left main coronary artery was cannulated with a JL4 6 Fr guide catheter; a 0.014 guidewire was introduced into the second septal branch and an over-the-wire (OTW) ballon catheter of $2.0 \times 9 \mathrm{~mm}$ was crossed through the guidewire placing it in the ostium of the septal branch and it was inflated to 14 atm (Figure 1). It was observed by transthoracic echocardiogram, 
a decrease of the interventricular gradient and of the contractility in the basal septal region after 10 minutes of balloon occlusion. Once confirmed by echocardiography that the area irrigated by the septal branch corresponded directly to the territory of hypertrophy, a $96 \%$ alcohol infusion was slowly initiated at a total of $3 \mathrm{ml}$. The OTW balloon catheter $2.0 \times 9 \mathrm{~mm}$ was inflated during the alcohol infusion to protect the anterior descending artery for 40 minutes. After ablation, immediate echography with akinesia in septal basal zone, as well as a decrease of the LVOT gradient of $30 \mathrm{~mm} \mathrm{Hg} \mathrm{mm} \mathrm{Hg}$; EKG with a $3 \mathrm{~mm} \mathrm{ST} \mathrm{segment} \mathrm{depression} \mathrm{in} \mathrm{the} \mathrm{anterior} \mathrm{face} \mathrm{and} \mathrm{ventricular}$ ectopy of left branch morphology (Figure 2). Contrast was injected to anterior descending artery with an adequate TIMI III flow, indicator of hemodynamic stability. Follow up at 6 months with improvement in functional class, NYHA I. Dobutamine stress echocardiogram with a dynamic LVOT gradient of $131 \mathrm{~mm} \mathrm{Hg}$ (Figure 3) right and left cavities of normal diameters and thickness, severe hypokinesia of the basal third of the anterior septum, LVEF $65 \%$, mild mitral valve SAM.
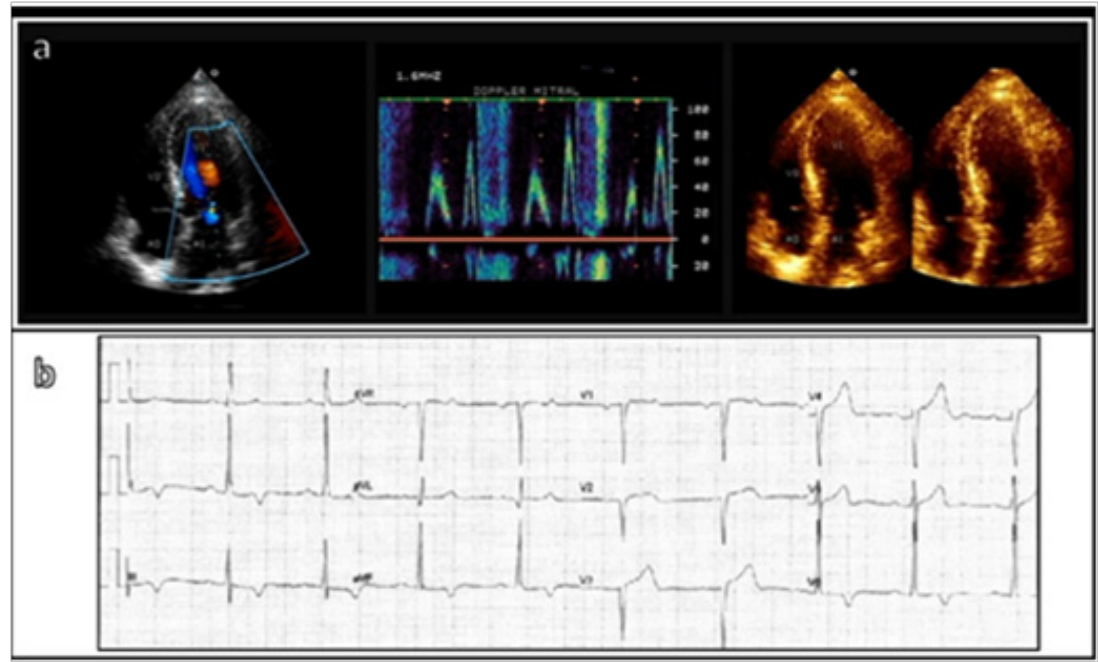

Figure I A) TT echocardiogram, B) Electrocardiogram with systolic overload.

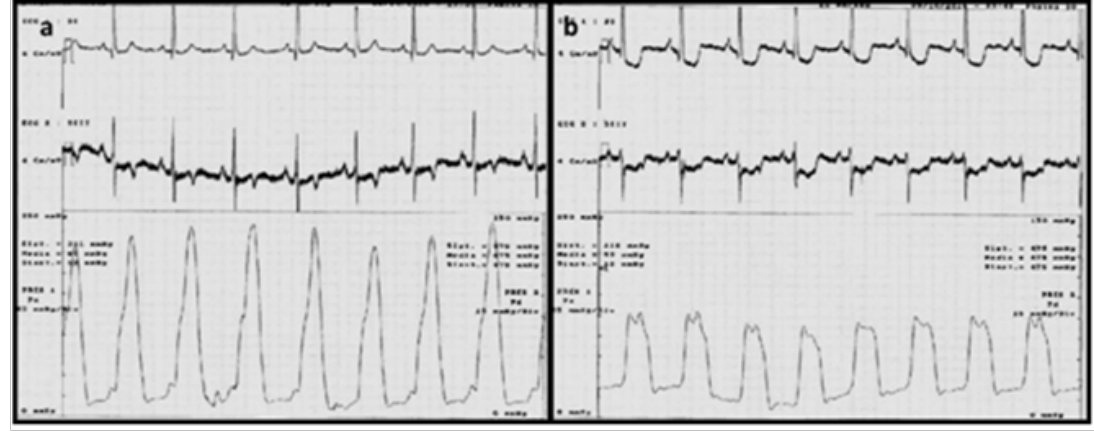

Figure 2 A) Pressure gradients and EKG before alcohol ablation, B) Pressure gradients and EKG after alcohol ablation.

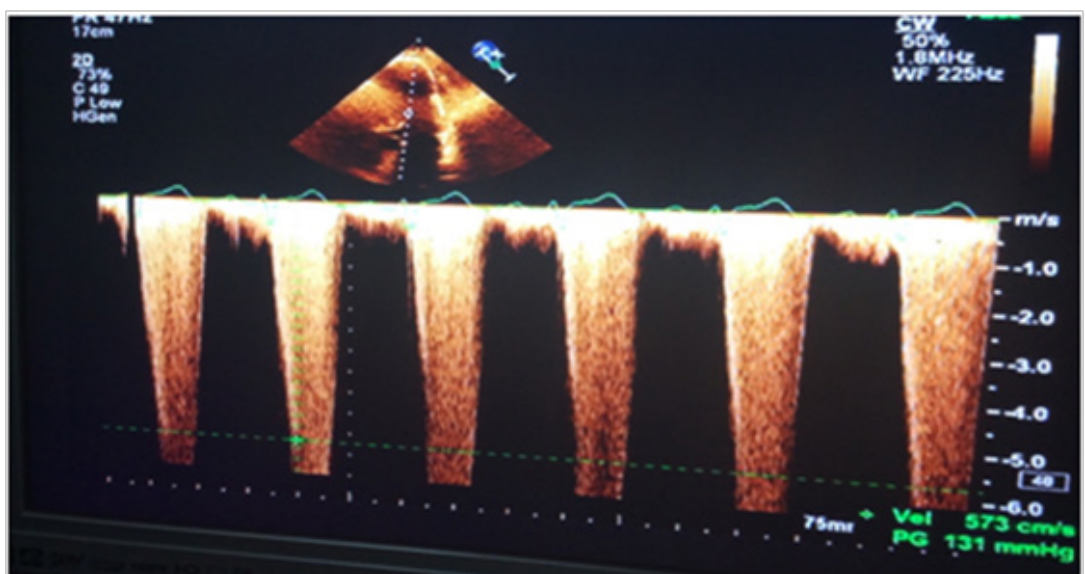

Figure 3 Dynamic LVOT gradient of $13 \mathrm{I} \mathrm{mm} \mathrm{Hg.}$ 


\section{Discussion}

ASA has merged as a percutaneous alternative to surgical myectomy. However, literature tends to support better long-term symptom relief in those patients who undergo septal myectomy, lower rate of complications, and immediate results over ASA with an immediate reduction in LVOT gradient. Also, patients with veru high LVOT gradients are considered poor candidates for $\mathrm{ASA}^{5}$ those patients with a very high LVOT gradient. According to the 2014 European Society of Cardiology (ESC) Guidelines, surgical myectomy is recommended in patients with a resting or maximum provoked LVOT gradient of $\geq 50 \mathrm{~mm} \mathrm{Hg}$, who are in NYHA functional class III-IV, despite maximum tolerated medical therapy (class of recommendation and level of evidence IB). ${ }^{2}$ Extremely high LVOT gradients $(>200 \mathrm{~mm} \mathrm{Hg})$ are uncommon, ${ }^{6}$ with several cases reported by Joshi et al., ${ }^{7}$ of $>200 \mathrm{~mm} \mathrm{Hg}$ and a case over $300 \mathrm{~mm} \mathrm{Hg}$ described echocardiographic records of patients undergoing dynamic changes of LVOT gradients ${ }^{7}$. However, to our knowledge this is the first documented case of a HCM with a very high dynamic LVOT gradient $>250 \mathrm{~mm} \mathrm{Hg}$ and an immediate reduction of resting gradient $>50 \%$ after ASA, and at 6 month follow-up reduction of dynamic LVOT gradient $>100 \mathrm{~mm} \mathrm{Hg}$; without complications during and after procedure, no development arrhythmogenic events during follow-up, improvement of NYHA functional class (NYHA I), and the expected hypokinesia in the site of septo-basal hypertrophy.

\section{Conclusion}

We present a unique case of HCM with a very high LVOT gradient and a successful management of obstructive gradient with ASA. We consider ASA as a safe and effective procedure in the reduction of very high LVOT in HCM.

\section{Acknowledgements}

None.

\section{Conflicts of interest}

The author declares there is no conflict of interest.

\section{References}

1. Ma H, Marti-Gutierrez N, Park S, et al. Correction of a pathogenic gene mutation in human embryos. Nature. 2017;548(7668):413-419.

2. Elliot PM, Anastasakis A, Borger MA, et al. 2014 ESC Guidelines on diagnosis and management of hypertrophic cardiomyopathy. Eur Heart J. 2014;35(39):2733-27779.

3. Liebregts M, Steggerda RC, Vriesendorp PA, et al. Long-Term Outcome of Alcohol Septal Ablation for Obstructive Hypertrophic Cardiomyopathy in the Young and the Elderly. JACC Cardiovasc Interv. 2016; 9(5):463-469.

4. Nagueh SF, Groves BM, Schwartz L, et al. Alcohol Septal Ablation for the Treatment of Hypertrophic Obstructive Cardiomyopathy. J Am Coll Cardiol. 2011;58(22):2322-2328.

5. Kotkar KD, Said SM, Dearani JA, et al. Hypertrophic obstructive cardiomyopathy: the Mayo Clinic experience. Ann Cardiothorac Surg. 2017;6(4):329-336.

6. Williams L, Gruner C, Rakowski H. Left Ventricular Outflow Tract Obstruction in Hypertrophic Cardiomyopathy: Do We Need to Stand to Be Counted. JASE. 2011;24(1):83-85.

7. Joshi S, Patel UK, Yao S, et al. Standing and Exercise Doppler Echocardiography in Obstructive Hypertrophic Cardiomyopathy: The Range of Gradients with Upright Activity. J Am Soc Echocardiogr. 2011;24(1):75-82. 